\title{
CT Diagnosis of Isolated Intramural Dissection of the Gallbladder Wall Following Blunt Abdominal Trauma
}

\author{
Benagiano G'*, Mori S ${ }^{2}$, Paolucci ML1, Semeraro \\ $A^{3}$, Colucci $F^{4}$, Dedola $G^{1}$ and Santini $S^{5}$ \\ ${ }^{1}$ Department of Radiology, San Giovanni di Dio Hospital, \\ Florence, Italy \\ ${ }^{2}$ Department of Surgery, San Giovanni di Dio Hospital, \\ Florence, Italy \\ ${ }^{3}$ Department of Radiology, Meyer Hospital, Italy \\ ${ }^{4}$ Department of Radiology, San Camillo Forlanini \\ Hospital, Rome, Italy \\ ${ }^{5}$ Department of Radiology, San Giuseppe Hospital, \\ Empoli, Italy
}

*Corresponding author: Benagiano G, Department of Radiology, San Giovanni di Dio Hospital, No 3 Torregalli Road, Post Code 50143, Florence, Italy

Received: July 03, 2017; Accepted: August 01, 2017; Published: August 11, 2017

\begin{abstract}
The gallbladder is rarely injured in blunt abdominal trauma due to its small size and anatomical protection by the surrounded liver, omentum and ribcage. Blunt gallbladder injuries are usually associated with damage of adjacent abdominal organs. Isolated gallbladder injury is an even more infrequent occurance. The diagnosis may be difficult and often delayed due to variable and non-specific clinical signs and symptoms, potentially increasing in the morbidity and mortality associated with traumatic gallbladder injuries. Prompt diagnosis is thus essential but represents a challenge. Of all imaging modalities, Computed Tomography (CT) is the most effective diagnostic tool for early identification of traumatic gallbladder injuries facilitating surgical planning and management. We present the first case of isolated intramural dissection of the gallbladder wall secondary to blunt abdominal trauma which was diagnosed by CT and subsequently confirmed by cholecystectomy.
\end{abstract}

Keywords: Gallbladder; Traumatic intramural dissection; Abdominal blunt trauma; Computed tomography (CT)

\section{Case Report}

A 48-year-old male with a history of chronic alcohol abuse, was admitted to the emergency department after crashing against a cemented wall with a car. On admission the patient referred right upper quadrant pain and was hemodynamic ally stable with blood pressure of $160 / 80 \mathrm{mmHg}$ and pulse rate of $73 / \mathrm{min}$. On physical exam there was right upper abdominal tenderness with positive Murphy's sign. Laboratory studies revealed a blood alcohol level of $2.28 \mathrm{~g} / \mathrm{L}$, alanine Aminotransferase (ALT) of $395 \mathrm{UI} / \mathrm{L}$, aspartate Aminotransferase (AST) of $630 \mathrm{UI} / \mathrm{L}$, Lactate Dehydrogenase (LDH) of $614 \mathrm{U} / \mathrm{L}$. Initial hemoglobin level was $14.9 \mathrm{~g} / \mathrm{dL}$. A FAST (Focused Assessment with Sonography in Trauma) examination revealed a distended gallbladder with wall thickened and pericholecystic fluid. Therefore a subsequent Computed Tomography (CT) was performed. Unhanced CT demonstrated marked enlargement of the gallbladder (measuring $15 \times 6 \times 7.5 \mathrm{~cm}$ ) with hyper dense intraluminal fluid compatible with blood (Figure 1A), estending into and distending the gallbladder neck and cystic duct. On contrast enhanced phase the gallbladder showed active arterial intraluminal extravasion, a site of mucosal discontinuity and a double-contour thickening of the anterior wall with evidence of a dissection flap (Figure 1B,C). Additionally a small amount of pericholecystic and perihepatic fluid was noted. Delayed images revealed an increased amount of intraluminal hyper density (Figure 1D) with fluid-fluid level as hemorrhage progress (Figure 1E). No evidence of other intraperitoneal injuries was noted. Therefore, the diagnosis of isolated gallbladder wall injury was considered based on imaging findings. After observation of 10 hours the patient complained of persistent right upper abdominal pain. Hemoglobin level decreased to $12.6 \mathrm{~g} / \mathrm{dL}$ and the onset of melena was noted. Exploratory laparotomy revealed a grossly enlarged gallbladder with intact serosa that showed a large subserosal ecchymosis on the anterior wall (Figure 2). A small amount of hemoperitoneum was present and no evidence of free bile in the peritoneal cavity and other abdominal injuries was observed. After abdominal wash, cholecystectomy was performed and an abdominal drain was placed. Postoperative course was uneventful and patient was discharged on the $8^{\text {th }}$ postoperative day. Upon opening the gallbladder postoperatively, an intramural hematoma between the layers of the anterior wall was present and the gallbladder lumen was full of fresh blood and blood clots. After removal of intraluminal hematoma, an about $2-\mathrm{cm}$ mucosal laceration of the anterior wall of the gallbladder and other small superficial mucosal tears were found (Figure 3). In conclusion, traumatic intramural dissection of the gallbladder wall with subsequent intramural hematoma was confirmed.

\section{Discussion}

The gallbladder is rarely injured in blunt abdominal trauma due to its small size and anatomical protection by the surrounded liver, omentum, intestine and ribcage [1-3]. Only $2 \%$ of patients undergoing laparotomy for blunt trauma show gallbladder injuries that are usually associated with damage of adjacent abdominal organs, especially the liver [1,3-7]. Consequently isolated blunt gallbladder injury is even more infrequent.

Most of blunt gallbladder injuries are caused by motor vehicles accidents (as a result of a crushing force or a seat belt compression) followed by injury from significant falls, kicks or blows to the abdomen [1,5,8]. Predisposing factors include a thin-walled or distended gallbladder such as in a fasting state and alcohol ingestion that increases sphincter of Oddi tone and biliary tract pressure resulting in gallbladder distension $[1,5,7,9]$.

Currently traumatic gallbladder injuries are classified as contusion, incomplete tear, laceration, avulsion and traumatic cholecystitis [2-4]. Contusion, defined as intramural hematoma, is often undiagnosed due to a lack of acute signs or symptoms [6]. 

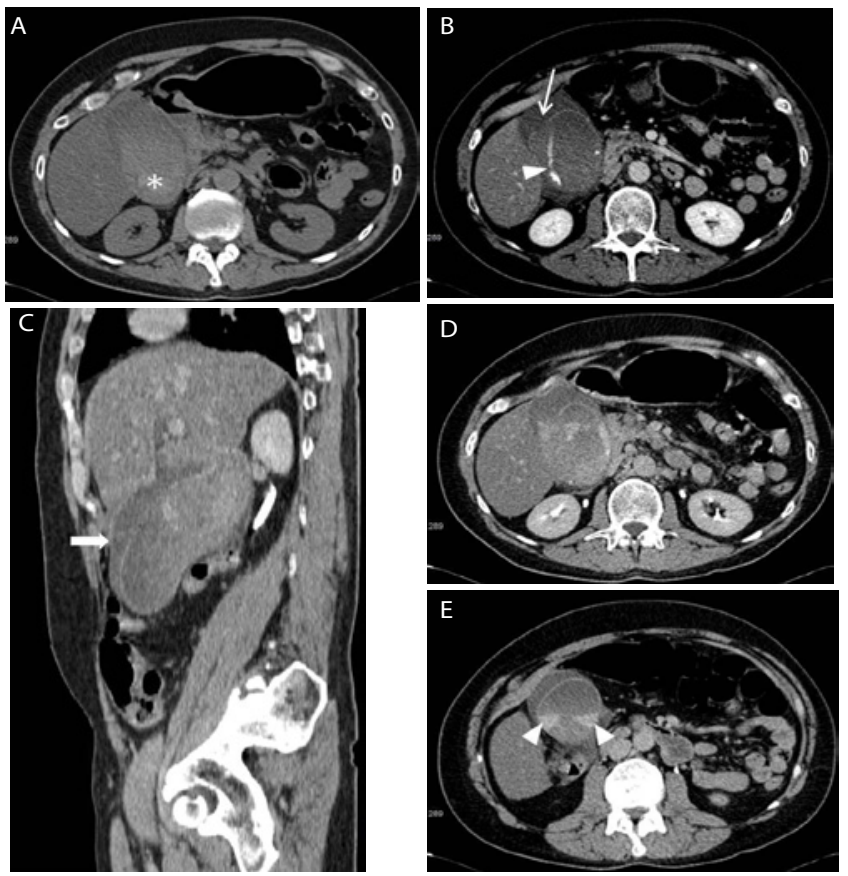

Figure 1: Intramural dissection of the gallbladder wall in a 48-year-old man after a motor vehicle accident. (A) Unhanced CT revealed hydropic gallbladder with hyper dense intraluminal fluid consistent of hemobilia (asterisk). (B) On axial contrast-enhanced CT the gallbladder shows active arterial intraluminal extravasion (arrowhead) and a site of mucosal discontinuity (arrow). (C) Coronal CT image demostrates double-contour wall thickness (arrow) with a dissection flap. (D, E) Delayed images revealed an increased amount of intraluminal hyperdensity with fluid-fluid level (arrowheads in E) as hemorrhage progress.

Incomplete tear consists in only mucosal tear $[3,4]$. Laceration, which includes rupture and perforation, is full-thickness wall injury and can result in leakage of bile [5,9-11]. Avulsion is sub classified as partial, complete, or total. Partial and complete avulsion injuries differ in the degree of separation of the gallbladder from the liver bed; in total avulsion, referred as traumatic cholecystectomy, the gallbladder is torn from all attachment. Traumatic cholecystitis is caused by cystic duct obstruction by blood clots from gallbladder injury [11].

In our case a tear on mucosa of the gallbladder bed allowed blood to enter the wall from the distended lumen with subsequent separation of the layers within the gallbladder wall. To our best knowledges this case represents the first reported occurance of intramural dissection of gallbladder wall with subsequent intramural hematoma following blunt abdominal trauma.

The clinical presentation of blunt gallbladder injury is variable and non-specific, and range from less common acute abdomen to slowly progressive abdominal complaints [5]. Consequently the diagnosis may be delayed and result in significant increase in morbidity or even mortality associated with traumatic gallbladder injuries [5]. Therefore early diagnosis is essential. CT is the most accurate and reliable imaging modality for early identification of traumatic gallbladder injuries facilitating surgical planning and management $[5,6,10]$.

Specific CT findings of traumatic gallbladder injuries involve the gallbladder wall and include: ill-defined contour or discontinuity of the gallbladder wall, active arterial intraluminal extravasation and a

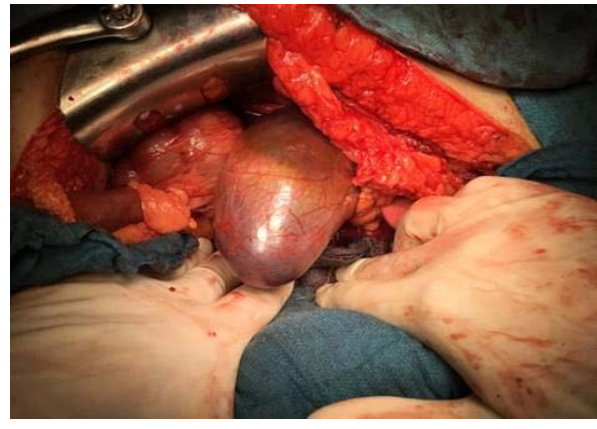

Figure 2: Hydropic gallbladder with diffuse subserosal ecchymosis on the anterior wall consistent of intramural hematoma.

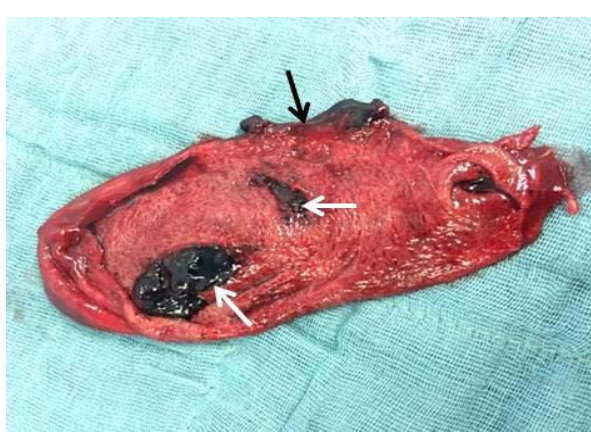

Figure 3: Mucosal laceration of the anterior wall (black arrow) and superficial mucosal tears of the gallbladder bed (white arrows).

site of mucosal distruption or non enhancement $[5-7,9,10,12]$.

The identification of high density material within the gallbladder lumen may be an indication of intraluminal hemorrhage (hemobilia) but it is a non-specific sign $[5,6]$. Cholelitiasis, hyper dense sludge, milk-of calcium bile and vicarious excretion of intravenous contrast material can mimic blood in the gallbladder lumen. However, delayed imaging can be useful in differentiating true gallbladder hemorrhage from other non traumatic gallbladder findings showing an increased amount of intraluminal hyper density or a fluid-fluid level as the hemorrhage progress $[5,9,10]$.

The finding of collapsed or unremarkable gallbladder, particularly in a fasting patient, should raise the possibility of gallbladder perforation or avulsion $[5,6,12]$.

Mural edema or thickening, pericholecystic fluid and mass effect on adjacent organs, are secondary signs that increase suspicion for the presence of gallbladder injury $[6,12]$.

In addition a contrast extravasion in pericholecystic area may indicate a vascular affection, tipically with arterial cystic avulsion [12].

The CT findings of hydropic gallbladder with intraluminal hemorrhage associated with wall injuries such as mucosal discontinuity, active arterial intraluminal extravasion and evidence of a dissection flap, indicate the diagnosis of intramural dissection of the gallbladder wall, as in our case.

The management depends on the severity of the gallbladder injury and general condition of the patient [10]. The treatment of severe injuries as rupture, perforation and complete or total avulsion 
is cholecystectomy as supported in literature [13]. Minor injuries can sometimes be observed although cholecystectomy can obviate the risk of delayed complications as traumatic cholecystitis and perforation that results from an intramural hematoma compromising the local blood supply to the wall [7].

The prognosis for blunt gallbladder injury, with early detection and lack of the other severe associated injury, remains quite good [14].

\section{References}

1. Sharma O. Blunt gallbladder injuries: presentation of twenty-two cases with review of the literature. J Trauma. 1993; 39: 576-580.

2. Soderstrom CA, Maekawa K, Dupriest RW Jr, Cowley RA. Gallbladder injuries resulting from blunt abdominal trauma: an experience and review. Ann Surg. 1981; 193: 60-66.

3. Kwan BYM, Plantinga $P$, Ross I. Isolated traumatic rupture of the gallbladder. Radiology Case Reports 2015; 10; 1029.

4. Losanoff JE, Kjossev KT. Complete traumatic avulsion of the gallbladder. Injury. 1999; 30: 365-368.

5. Wittenberg A, Minotti AJ. CT diagnosis of traumatic gallbladder injury. AJR Am J Roentgenol. 2005; 185: 1573-1574.

6. Cheng YC, Yen PC, Tsai IT. Isolated Gallbladder Injury Due to Blunt. Abdominal Trauma: A Case Report. E-Da Medical Journal. 2014; 1: 35-37.
7. Chen X, Talner LB, Jurkovich GJ. Gallbladder avulsion due to blunt trauma. AJR. 2001; 177: 822.

8. Sondenaa K, Horn A, Nedrebo T. Diagnosis of blunt trauma to the gallbladder and bile ducts. Eur J Surg. 2000; 166: 903-907.

9. Mohanty D, Agarwal H, Aggarwal K, Garg PK. Delayed rupture of the gallbladder following blunt abdominal trauma. Maedica - a journal of clinical medicine. 2014; 9: 266-268.

10. Nishiwaki M, Ashida H, Nishimura T, Kimura M, Yagyu R, Nishioka A, et al. Posttraumatic intra-gallbladder hemorrhage in a patient with liver cirrhosis. J Gastroenterology 1999; 34: 282-285

11. Birn J, Jung M, Dearing M. Isolated gallbladder injury in a case of blunt abdominal trauma. J Radiol Case Rep. 2012; 6: 25-30.

12. Gupta A, Stuhlfaut JW, Fleming KW, Lucey BC, Soto JA. Blunt trauma of the pancreas and biliary tract: a multimodality imaging approach to diagnosis. Radiographics. 2004; 24: 1381-1395.

13. Yeung KW, Liu PP. Isolated Gallbladder Perforation Following Blunt Abdominal Trauma: a case report. J Radiol Sci. 2014; 39: 51-55.

14. Ball CG, Dixon E, Kirkpatrick AW, Sutherland FR, Laupland KB, Feliciano DV. A decade of experience with injuries to the Gallbaldder. J Trauma Manag Outcomes 2010; 4: 3 .
Austin J Radiol - Volume 4 Issue 2 - 2017

ISSN : 2473-0637 | www.austinpublishinggroup.com

Benagiano et al. (C) All rights are reserved
Citation: Benagiano G, Mori S, Paolucci ML, Semeraro A, Colucci F, Dedola GL, et al. CT Diagnosis of Isolated Intramural Dissection of the Gallbladder Wall Following Blunt Abdominal Trauma. Austin J Radiol. 2017; 4(2): 1068. 